\title{
Herding, A-synchronous Updating and Heterogeneity in Memory in a CBS*
}

\author{
Cees Diks \& Roy van der Weide
}

CeNDEF, Faculty of Economics and Econometrics, University of Amsterdam, and

Tinbergen Institute, Roetersstraat 11, 1018 WB, Amsterdam, The Netherlands

November 4, 2003

\begin{abstract}
This paper considers a simple Continuous Beliefs System (CBS) to investigate the effects on price dynamics of several behavioral assumptions: (i) herd behaviour; (ii) a-synchronous updating of beliefs; and (iii) heterogeneity in time horizons (memory) among agents. The recently introduced concept of a CBS allows one to model the co-evolution of prices and the beliefs distribution explicitly, while keeping track of the unpredictable nature of individual preferences (Diks and van der Weide, 2003). As a benchmark model we take a simple CBS, which in a market with many traders exhibits a random walk driven by news. Using the explicit nature of the dynamics of the CBS we show that the introduction of herding modifies the random walk to an $\operatorname{ARIMA}(0,1,1)$ process, which is observationally equivalent to a reduction of the number of market participants. In terms of returns the model predicts MA(1) structure with a negative coeffient. Asynchronous updating leads to an MA(1) model for returns with $\operatorname{GARCH}(1,1)$ innovations, and predicts a relation between the ARCH and GARCH coefficients. Heterogeneity in memory leads to long-range dependence in returns. In the empirical section we perform a modest 'reality check' concerning the predicted sign of the MA coefficient and the relation between the ARCH and GARCH coefficients for exchange rate data.
\end{abstract}

Keywords: Continuous beliefs systems; Random dynamical systems; Heterogeneity; Herding; ARIMA; GARCH; Long-range dependence;

JEL classification: C5, G1, D8, F31

* Research supported by the Netherlands Organization for Scientific Research (NWO) under a MaGW-Pionier and a MaGW-Vernieuwingsimpuls grant. 


\section{Introduction}

The current literature on agent based economic models can be roughly divided into two types: (i) computational finance models and (ii) economic dynamic models. Both approaches aim to discover what is behind the stylized facts commonly exhibited by empirical financial time series.

In computational finance, artificial stock markets are used as a tool for simulating time series of aggregate variables, such as prices, volumes, etcetera (for an overview, see LeBaron, 2000). To improve the understanding of markets with a large number of interacting heterogeneous agents, LeBaron et al. (1999) for example developed the Santa Fe artificial stock market. In the Santa Fe market, the actions of agents, which are based upon their expectations, are explicitly modelled and traced for each individual agent. With computers becoming cheaply available and faster, these artificial markets allow for more and more detailed modelling. Although detailed models may possibly generate more realistic price dynamics, their increasing complexity also makes simulation less attractive as a tool for the analytic study of the mechanisms linking the heterogeneity of beliefs with the stylized facts of prices.

The economic dynamics literature follows a somewhat different route. There agent based modelling explicitly aims at an analysis of the joint dynamics of beliefs and prices (see e.g. Brock and Hommes, 1997, 1998). To achieve the necessary analytical tractability, several simplifying assumptions are usually made, such as a continuum of agents who at each time can choose from a finite number of belief types. Typically a low dimensional deterministic dynamical system is derived, allowing the modeller to perform a detailed bifurcation analysis, providing insights in the long-run behaviour of the dynamics for different economic and behavioral parameters. The natural tradeoff of this approach is that it at best provides an approximation to the deterministic aspects of the price process of interest. Indeed, the 'holy grail' in economic dynamics is beyond any doubt the heterogeneous agent model that is just as realistic in terms of its price dynamics as it is tractable analytically.

In previous work (Diks and van der Weide, 2003) we introduced the concept of a continuous beliefs system (CBS). This framework is built around a continuous beliefs space representing the possible point predictors agents can choose from. On this space a time dependent beliefs distribution is defined, which is updated according to a continuous choice model. As new market prices become available, the beliefs distribution is updated depending on past performances of strategies. Coevolution of the beliefs distribution with price dynamics thus arises as newly realized prices feed the ongoing evaluation of the strategies by the agents. As is natural in a continuous choice setting, individual choices are considered to be random variables, distributed according to the beliefs distribution. The preferences of agents provide an endogenous noise source which affects price dynamics. Because the randomness in prices can be associated naturally with the diversity of beliefs, the latter has been labelled a 'natural source of randomness'.

Diks and van der Weide (2003) discuss several scenarios under which the natu- 
ral randomness in prices does not average out in the limit as the number of agents tends to infinity. These include: (i) a disproportionate distribution of market impacts; (ii) particular combinations of utility functions and predictors, and (iii) dependence among agents, which may arise for example when agents partly coordinate on a random variable, not necessarily related to economic fundamentals ('sunspots'). All these examples are based on a violation of at least one of the conditions of the law of large numbers. In the last case (dependence) it is often impossible to distinghuish between an infinite number of dependent agents, or a finite number of independent agents. For convenience we therefore introduced the notion of an effective number of agents, $n_{\text {eff }}$, defined as the number of independent agents required to obtain stochastic price dynamics with similar stochastic dynamics. For a finite effective number of agents the dynamics is random by construction. In general, a CBS gives rise to a random dynamical system, allowing for the stochastic price dynamics to be described explicitly. It therefore offers an analytic alternative to the simulation based studies commonly performed in computational finance.

In sum, a CBS shares several advantages of both computational finance models and economic dynamic models. Rather than obtaining the stochastic macroscopic dynamics through laborious simulation, the macroscopic behaviour of a CBS with agents who can choose among a continuum of alternative strategies, can often be derived analytically. The aggregate behaviour can typically be captured in a few simple dynamic equations, for an arbitrary number of agents. The analytic tractability can be used to study features such as the conditional mean and variance of prices analytically, and relate these to behavioural characteristics.

The objective of this paper is to examine the effects on price dynamics of a number of behavioral assumptions. We introduce a simple benchmark CBS and expand it in the following directions: (i) herd behavior; (ii) a-synchronous updating of beliefs; and (iii) heterogeneity in the memory of agents. The behavioral models obtained in this way give rise to aggregate dynamics which are represented by wellknown classes of econometric models. At the center is the random walk obtained for our benchmark CBS. We will show that that: (i) herd behaviour introduces moving average (MA) structure yielding an ARIMA model; (ii) an ARIMA model with GARCH disturbances is obtained if beliefs are not updated synchronously; and (iii) a fractionally integrated (ARFIMA) process with long-range dependence is obtained if the memory of agents exhibits heterogeneity. As an immediate result the parameters of these standard econometric models can be related directly to the parameters of the behavioral model.

Near the end of the paper we will only carefully scratch the surface of the possible empirical applications. This is done by estimating some of the stylized models using daily exchange rate data. We wish to emphasize honestly at the outset, however, that while interpreting the empirical results in terms of behavioral parameters, one should keep in mind that the models are stylized and, for clarity of exposition, deliberately stripped from a range of features that may become important for a realistic summary of the data. For example, we are aware of the fact that our natural source of random- 
ness is not the only relevant source of randomness, and that more factors than those included in our behavioral model may be responsible for MA and/or GARCH structure in the empirical data. Although omitting additional relevant model aspects will surely bias our parameter estimates, we consider comparing the signs of the estimated parameters with those expected from our theory a sensible 'reality check'. If successful, such an empirical validation may hopefully open doors to more detailed empirical assessments in the future.

Finally note that inherent in our approach is the assumption that the agents believe in structure in prices that can be profitably exploited by 'technical trading rules' summarized in terms of point predictors, which we will refer to interchangeably as strategies or beliefs. Empirical evidence supporting this belief in predictability in the foreign exchange markets is provided by e.g. Sweeney (1986), Taylor and Allen (1992), Levich and Thomas (1993) and LeBaron (1999). Furthermore, our approach is entirely built around the heterogeneity of beliefs. Some arguments for expectations being heterogeneous have been provided by, among others, Frankel and Froot (1990). In a theoretical exchange rate model, Frankel and Froot (1988) particularly employ the heterogeneity of expectations to explain some of their empirical findings.

In the following section we briefly review the basic concepts of a CBS. The introduction of the benchmark CBS and its extensions are described in section 3. In section 4 the models will be subjected to a basic empirical validation. Section 5 concludes.

\section{Continuous beliefs systems}

In this section we briefly review the concept of a continuous beliefs system. The main ingredients for describing the co-evolution of prices and the beliefs distribution in a CBS are the following. As is common in economic dynamics models, agents are assumed to predict future prices by using a point predictor which is a function of the information available to them (we typically have in mind past realized prices). The point predictors agents can choose from are represented in a beliefs space $\Omega$, parameterized by a continuous parameter $\theta$, called the belief parameter. Each value of $\theta \in \Omega$ represents a predictor $x_{t+1}^{e}(\theta)$ of the next price in terms of the information $\mathcal{F}_{t}$ available to the agents. For example if agents believe in a linear world and expect tomorrow's price $x_{t+1}$ to be a linear function $x_{t+1}^{e}(\theta)=\theta_{0}+\theta_{1} x_{t}+\ldots+\theta_{d} x_{t-d+1}$ of the last $d$ prices, then the beliefs space consists of the $d+1$-dimensional Euclidean space $\mathbf{R}^{d+1}$.

Given the individual beliefs $\left\{\theta_{i, t-1}\right\}$, a realization of the price $x_{t}$ is obtained via some market clearing mechanism. Agents' utility functions and predictors imply individual demand functions, and the market price is defined to be the unique price $x_{t}$ for which the market clears. ${ }^{1}$ Of course the appropriate market clearing price equation depends

\footnotetext{
${ }^{1}$ Uniqueness of market clearing prices is not self-evident. However, if demands are strictly decreasing in $x_{t}$, as will be the case in our benchmark CBS, uniqueness of clearing prices is guaranteed.
} 
on the context, and we will have to provide some additional arguments to justify our particular choice made for the benchmark model later.

As new prices become available, agents update their evaluations of parameter values by confronting them with the newly arrived information. As agents have differences in tastes and interpret identical information differently they will typically choose different predictors. This is captured by a continuous choice model, which defines a probability distribution on the beliefs space $\Omega$. The probability density function of this time dependent beliefs distribution is denoted by $\phi_{t}(\theta)$. The actual belief parameters $\theta_{i, t}$ individual agents use to base their next prediction on are modelled as random variables distributed according to $\phi_{t}(\theta)$. In this way endogenous randomness enters the price dynamics in a natural way. In the simplest case the $\theta_{i, t}$ are assumed to be independent conditional on information available up to and including time $t$.

To see how the beliefs distribution evolves, let us consider the time when $x_{t}$ has just been quoted. After $x_{t}$ becomes public, agents re-evaluate the strategies using a performance measure, $\pi_{t}(\theta)$. The performance depends on past realized prices and might for example be based on ex post prediction errors, or the ex post profits realized by a strategy. A typical choice is to take $\pi_{t}(\theta)$ to consist of minus the ex post squared prediction error:

$$
\pi_{t}(\theta)=-\left(x_{t}^{e}(\theta)-x_{t}\right)^{2} .
$$

This choice is less restrictive as it may seem, since for $\phi_{t}(\theta)$ only differences in performance play a role. Using this property it can be shown that minus squared prediction errors are equivalent to risk adjusted profits when agents hold identical beliefs about conditional variances (see e.g. Hommes, 2001). In practice, decisions to change strategies are not based on yesterday's performances only, but rather on the success over a larger history. Indeed, financial analysts typically test their candidates on a large sample of past price history, also known as back-testing. Strategies that have performed best over some history of the sample are more likely to be selected for future trading. The simplest way to implement, or mimic, this back-testing is to consider a geometrically down-weighted average of past performances rather than only the last performance, so that the weighted average utility becomes:

$$
U_{t}(\theta)=(1-\alpha) \sum_{i=0}^{\infty} \alpha^{i} \pi_{t-i}(\theta),
$$

which can be recast into the more practical form

$$
U_{t}(\theta)=\alpha U_{t-1}(\theta)+(1-\alpha) \pi_{t}(\theta) .
$$

Given the average past utility the continuous logit model provides the following pdf for the beliefs distribution just after time $t:^{2}$

$$
\phi_{t}(\theta)=\frac{\mathrm{e}^{\beta U_{t}(\theta)}}{Z_{t}},
$$

\footnotetext{
${ }^{2}$ In Diks and van der Weide (2003) the right-hand-side was multiplied by a so-called opportunity function $\varphi(\theta)$ which we take equal to unity here.
} 
where $Z_{t}$ is a normalization constant independent of $\theta$. The parameter $\beta$ is referred to as the intensity of choice. The inverse $1 / \beta$ is also known as the propensity to err. If agents are sensitive to small differences in utility, this will be reflected by a large value of $\beta$. Notice that Eq. (3) implies that only differences in performance play a role. Modifications of $\pi_{t}(\theta)$ that are independent of $\theta$ will be absorbed by the normalization factor $Z_{t}$, leaving $\phi_{t}(\theta)$ unchanged. With this in mind it is not surprising that risk adjusted profits and minus squared prediction errors turn out to be equivalent under certain conditions.

Substitution of Eq. (2) into Eq. (3) gives

$$
\phi_{t}(\theta)=\frac{\mathrm{e}^{\alpha \beta U_{t-1}(\theta)+(1-\alpha) \beta \pi_{t}(\theta)}}{Z_{t}}=\frac{\left[\phi_{t-1}(\theta)\right]^{\alpha} \mathrm{e}^{(1-\alpha) \beta \pi_{t}(\theta)}}{Z_{t}^{\prime}},
$$

which expresses how a new beliefs distribution can be obtained from the previous beliefs distribution and the last performance measure. The variable $Z_{t}^{\prime}$ is again a normalization constant. Although $\phi_{t}(\theta)$ is an infinite dimensional state variable, the time evolution of $\phi_{t}(\theta)$ can often be fully specified by a finite number of variables such as its first $k$ moments, in which case the dynamics becomes finite dimensional. In summary, the expectations feedback can be represented schematically as

$$
\ldots x_{t-1} \rightarrow \phi_{t-1}(\theta) \leadsto x_{t} \rightarrow \phi_{t}(\theta) \leadsto x_{t+1} \rightarrow \ldots,
$$

where “ $\rightarrow$ " indicates a deterministic step and “ $\rightarrow$ " a step involving endogenous noise.

It is important to note that the beliefs distribution $\phi_{t}(\theta)$ is determined only by the performance measure and past realised prices, and otherwise evolves independently of the individual choices of agents. The beliefs distribution is a convenient concept introduced in the choice literature to model the fact that agents have idiosyncrasies in tastes, and that their choices are not identical given identical information. The fact that identical information is interpreted differently by agents is confirmed empirically by Kandel and Pearson (1995). The strategies $\theta_{i, t}$ used by the agents, from the point of view of the econometrician, are random variables, representing the individual choices of agents, so that $x_{t+1}$ is a stochastic variable, even in the absence of exogenous shocks.

\section{A simple benchmark CBS and its extensions}

In this section we introduce a benchmark CBS and examine the effects on the dynamics of extending the model in the following behavioral directions: (i) herding, (ii) asynchronous updating, and (iii) heterogeneity in memory. The CBS formulation enables one to obtain insights into how these behavioral assumptions affect the interaction between the stylized facts and the unobserved beliefs distribution. The benchmark model is an extremely simple CBS deliberately stripped from any features that are not of direct interest. Although taking such a highly stylized model as a reference point 
may seem overly restricted, it clarifies the exposition and helps to isolate the effects of each of the extensions.

We wish our benchmark CBS to be as simple as possible while preserving economic relevance and bringing out the essentials of the CBS concept. Typically a CBS will be used for modelling the joint dynamics of beliefs and prices of well-specified economic units, such as shares, which are traded sequentially in a large market. Prices in such markets are determined by the expectations held by agents regarding future prices and cash flows, as well as their preferences concerning returns and risk. Agents interact by submitting demand functions depending on expected utility, while their realized utility is determined by realized market clearing prices. Regardless of preferences, it is clear that better abilities to predict future prices given the information available will help agents in realizing a higher utility. In this sense the setting resembles an ever ongoing version of Keynes' beauty contest.

Motivated by these arguments we consider a very stylized market mechanism which assumes prices to equal the average prediction over all agents in the market, i.e. the market expectation:

$$
x_{t}=\frac{1}{n} \sum_{i=1}^{n} x_{t+1}^{e}\left(\theta_{i, t-1}\right)
$$

where $n$ denotes the number of agents. Price equations used in economic dynamic models often involve similar market expectations. Depending on the type of market, also the risk free interest rate and expectations concerning dividend payments may enter the price equation. We have in mind agents who are myopic in the sense that they are concerned only with maximizing their one-step-ahead utility. At each time then, agents act as if they are entering the market today while knowing that they will leave the market tomorrow. As a result, only their expectations concerning the next price and additional cash flows (such as dividends) play a role. Typically, dividend payments (if any) are rare and announced well in advance, which means that expectations regarding short run profits are driven by expectations concerning future prices, rather than dividends. If, moreover, time intervals are sufficiently short (e.g. one to five days, see Lehmann, 1991) the interest rates over a single interval are sufficiently small for agents to consider their predicted future price to be a 'fair price' today as well. Under those conditions, if we assume agents to be mean-variance optimizers the market clearing price is given by the right hand side of Eq. (5). ${ }^{3}$

The simplest continuous class of point predictors consists of a continuous range of different constants. We incorporate this by saying that agents with belief $\theta$ predict

\footnotetext{
${ }^{3} \mathrm{~A}$ mean-variance optimizing agent with belief $\theta$ expects a risk-adjusted short-term profit of $d_{t}\left[x_{t+1}^{e}(\theta)-x_{t}\right]-\frac{1}{2} c d_{t}^{2}$, where $d_{t}$ is his demand and $c$ is a risk aversion parameter times the perceived conditional variance of $x_{t+1}$. Maximizing this with respect to demand $d_{t}$, gives the demand function $d_{t}=\left(x_{t+1}^{e}(\theta)-x_{t}\right) / c$. If $c$ is homogeneous among agents, the unique equilibrium price is given by Eq. (5). Heterogeneity in risk aversion and or perceived risk would lead to a weighted average $x_{t}=\sum w_{i} x_{t+1}^{e}\left(\theta_{i, t+1}\right) / \sum w_{i}$ with weights $w_{i}=c_{i}^{-1}$, which in a large market is equivalent to Eq. (5).
} 
tomorrow's price to be equal to $\theta$ :

$$
x_{t+1}^{e}(\theta)=\theta .
$$

Heterogeneity will arise if agents employ different beliefs in that they have in mind different parameter values $\theta_{i, t}$. The conditional variance $\operatorname{Var}_{t}\left[\theta_{i, t}\right]$ can then be considered to be a measure for the degree of heterogeneity. Forecast surveys of foreign exchange market participants, for example, indicate that such a dispersion of beliefs indeed exists, as found by Frankel and Froot (1990). An argument they provide in favour of heterogeneity is the high trading volume observed in the foreign exchange market, since it takes differences of opinion to trade.

For the performance measure we take minus squared prediction errors (Eq. 1), and memory is included in the utility function through Eq. (2). Having observed and compared overall performance, all agents subsequently adapt their beliefs. The new distribution of beliefs is determined by means of the continuous choice model, and is given by Eq. (3). Because the utility function is a quadratic function in the belief parameter $\theta$, it follows that, for all $t$, the distribution of beliefs is described by a normal distribution:

$$
\phi_{t}(\theta)=\frac{1}{\sqrt{2 \pi} \sigma_{t}} \exp \left[-\frac{1}{2}\left(\frac{\theta-\mu_{t}}{\sigma_{t}}\right)^{2}\right] .
$$

Normality implies that the beliefs distribution is fully specified by its mean $\mu_{t}$ and variance $\sigma_{t}^{2}$. Using Eq. (4) it can be seen that the dynamics of $\mu_{t}$ and $\sigma_{t}$ is governed by:

$$
\begin{aligned}
\mu_{t} & =\alpha\left(\frac{\sigma_{t}^{2}}{\sigma_{t-1}^{2}}\right) \mu_{t-1}+2(1-\alpha) \beta \sigma_{t}^{2} x_{t} \\
\frac{1}{\sigma_{t}^{2}} & =\frac{\alpha}{\sigma_{t-1}^{2}}+2(1-\alpha) \beta .
\end{aligned}
$$

In the long run $\sigma_{t}^{2}$ will converge to $\sigma^{2}=\frac{1}{2 \beta}$, and the dispersion of beliefs thus tends to a constant proportional to the propensity to err. If the propensity to err is high, agents are less sensitive to differences in performances of strategies. As a consequence, a large propensity to err implies a larger dispersion of beliefs. If we substitute $\sigma^{2}=\frac{1}{2 \beta}$ into the dynamics for the average belief $\mu_{t}$, we obtain:

$$
\mu_{t}=\alpha \mu_{t-1}+(1-\alpha) x_{t} .
$$

Having derived the dynamics for beliefs, we now shift focus to its interaction with the price equation Eq. (5) which in the constant predictor case becomes:

$$
x_{t}=\frac{1}{n} \sum_{i=1}^{n} x_{t}^{e}\left(\theta_{i, t-1}\right)=\frac{1}{n} \sum_{i=1}^{n} \theta_{i, t-1},
$$

For simplicity, we consider the system in the limit as the number of agents tends to infinity. In the absence of dependence among agents, the law of large numbers applies, 
and it follows that the observed price quoted as the average prediction will converge to $\mu_{t-1}$ :

$$
x_{t}=\lim _{n \rightarrow \infty} \frac{1}{n} \sum_{i=1}^{n} \theta_{i, t-1}=E\left[\theta_{i, t-1}\right]=\mu_{t-1} .
$$

Together with the 'dynamics' of the mean $\mu_{t}$ described by Eq. (8) this gives:

$$
x_{t}=\alpha x_{t-1}+(1-\alpha) x_{t-1}=x_{t-1} .
$$

Thus in the absence of uncertainty, the price will be constant at all times.

Exogenous shocks, such as news, are assumed to affect the price equation only through the expectations of agents. This assumption appears natural, since the only way news can possibly affect prices is indeed through expectations of individuals. The dynamics become

$$
\begin{aligned}
& x_{t}=\mu_{t-1}, \\
& \mu_{t}=\alpha \mu_{t-1}+(1-\alpha) x_{t}+\xi_{t+1},
\end{aligned}
$$

where $\xi_{t+1}$ is a noise term representing news shocks arriving between time $t$ and $t+1$. Upon substituting the first of those equations in the second, it can be seen that $\mu_{t}$ follows a random walk driven by $\xi_{t}$. The first equation states that $x_{t}$ simply equals $\mu_{t-1}$, the average belief under agents given all observables up to and including time $t-1$, and the news that has become available before time $t{ }^{4}$ In terms of increments we thus have, in the presence of exogenous shocks $\left\{\xi_{t}\right\}$ :

$$
\Delta x_{t} \equiv x_{t}-x_{t-1}=\xi_{t} .
$$

The random walk model is consistent with the efficient market hypothesis (EMH) and has been traditionally viewed as the model for financial variables, such as asset prices and exchange rates. Indeed, the random walk has long been considered unbeatable, a view epitomized by Malkiel (1990). Only recently it seems that it has to give in to a growing list of statistical evidence against the EMH, some of which is collected in Lo and MacKinlay (1997). As a consequence we see that more sophisticated models have begun to make their appearances. Kilian and Taylor (2001), for example, introduced an exchange rate model which is close to a unit root process near the fundamental, but in which the exchange rate is driven back to the fundamental when their difference becomes too large. They report empirical evidence supporting predictability of exchange rates at long horizons, but at short horizons exchange rates are hard to distinguish from a random walk.

\footnotetext{
${ }^{4}$ The random walk price dynamics can alternatively be derived with agents forming expectations of the type $x_{t}^{e}(\theta)=R_{t}+\theta$, where $R_{t}$ is a publicly known estimate of the fundamental exchange rate with increments $R_{t}-R_{t-1}=\xi_{t}$. In that case $\mu_{t}$ is stationary, while the exchange rate follows a random walk, $x_{t}=R_{t}+$ cnst.
} 


\subsection{Herd behaviour}

If the conditions for the law of large numbers (LLN) are not satisfied, the price dynamics may be stochastic in a large (many trader) market even in the absence of exogenous uncertainty. In the introduction we briefly mentioned a number of such 'sources of randomness' that might cause the LLN to fail. These are addressed in more detail in Diks and van der Weide (2003). Here we will focus on the dependence among agents arising from herd behaviour, as a source of 'natural randomness'. It turns out that sufficient correlation between the agents will indeed violate the conditions for the LLN to apply.

In the literature many different definitions of herding have been described. The type of herding we wish to address is that where conditional on public information, the strategies used by the agents exhibit dependence. In particular we have in mind situations where agents herd to publically announced opinions of others. During each period some 'early' agents reveal their expectations, allowing other agents to adapt their expectations towards those of the 'leaders' before submitting their market orders. This type of herding is 'concurrent' in that it involves agents coordinating simultaneously on common signals. Concurrent herding is different from studies that associate herding with for example informational cascades or learning (see e.g. Bikhchandani et al., 1992; Avery and Zemsky, 1998 and Nelson, 2002). In informational cascades uninformed agents try to mimic, or learn from, the more informed by extracting information from realized prices. This type of herding therefore has a 'sequential' nature. As one would expect, having in mind 'concurrent' or 'sequential' herding makes a difference. For example, Nelson (2002) finds that 'high correlation among agents' actions does not necessarily imply herding'. As in our model dependence among actions must originate from dependence in strategies, we would refer to such correlation among actions as herding by definition.

Possible motivations for (concurrent) herding are numerous. First note that according to Kandel and Pearson (1995), even though each individual typically has his own interpretation of public signals only few behave completely independent. A motivation is that being too different from the rest can be risky and might jeopardize career perspectives or reputation (see e.g. Trueman, 1994 and Scharfstein and Stein, 1990). Hong et al. (2000) have recently strengthened these arguments by providing some empirical evidence in favour of them. When delving into experience, they also find that younger analysts forecast closer to the average forecast, and that they are more likely to be terminated when they deviate from the consensus.

Even when accurate (short term) forecasting is the only objective agents have, herding might still be rational. Scharfstein and Stein (1990), as an oblique remark not that: "...investing is also driven by group psychology, which weakens the link between information and market outcomes." Indeed, note that in our benchmark CBS prices are entirely driven by speculation (group psychology). ${ }^{5}$ Froot et al. (1992) have given the

\footnotetext{
${ }^{5} \operatorname{Lux}$ (1995) is another paper that considers the possibility where agents pay more attention to each other than to the fundamentals. He assumes a market in which initially only optimists and pessimists
} 
thought more attention, and concluded the following. In the short term, speculative forces may be stronger than those of the 'underlying economic system'. As a consequence, price series generally show fluctuations around the fundamental, where one can always find short periods in time where prices exhibit divergence. In such periods it is thus rational to coordinate on speculative behaviour (herding) rather than on fundamentals.

Kirman (1993) describes herding as arising from a process, which involves agents that make choices first, 'recruit' other agents to make the same decision. There are several possible forms in which this recruiting takes place, which we will not delve into here. What is important for our model is that there may be mechanisms that introduce dependence among the strategies selected by the agents. The 'recruiting' scenario is an example mechanism which gives just this type of dependence in our model.

Herding towards a leading agent can be implemented easily in a CBS by introducing interaction terms into the utility function. ${ }^{6}$ For simplicity we assume that there is only a single leading agent. Let $\theta_{0, t}$ denote the parameter value announced publically by the leading agent, which we assume to be distributed according to the original beliefs distribution given by Eq. (6). Upon taking a new utility function $\tilde{U}_{t}(\theta)=$ $U_{t}(\theta)-J\left(\theta-\theta_{0, t}\right)^{2}$, the beliefs distribution of the other agents becomes

$$
\phi_{t}(\theta)=Z_{t}^{-1} \exp \left[\beta U_{t}(\theta)-\beta J\left(\theta-\theta_{0, t}\right)^{2}\right] .
$$

The interaction strength $J>0$ can be considered as a measure for the desire of agents to select a parameter value close to that of the leader. Straightforward comparison of coefficients of powers of $\theta$ in the exponent with those in Eq. (6) gives the following values for the mean $\tilde{\mu}_{t}$ and variance $\tilde{\sigma}_{t}^{2}$ of the beliefs distribution under herding:

$$
\tilde{\mu}_{t}=\mu_{t}+\frac{J\left(\mu_{t}-\theta_{0, t}\right)}{J+1} \equiv \mu_{t}
$$

and

$$
\tilde{\sigma}^{2}=\frac{\sigma^{2}}{1+\sigma^{2} J} .
$$

In the latter relation the time indices have been dropped as $\sigma_{t}^{2}$ was already known to be constant over time (see Eq. (7) and the discussion thereafter).

Under the assumption that market weights of individual agents (including the leading agent's) are negligible, realized prices are given by the mean of the pdf in Eq. (11):

$$
x_{t}=\tilde{\mu}_{t-1}=\mu_{t-1}+\sigma \lambda u_{t}
$$

where $\left\{u_{t}\right\} \equiv\left\{\left(\mu_{t-1}-\theta_{0, t-1}\right) / \sigma\right\}$ is a sequence of independent standard normal random variables.

can be distinguished. The evolution of the number of optimists is completely determined by the numbers of optimists and pessimists that surround individuals. At a later stage he assumes the presence of a fundamentalist.

${ }^{6}$ See Diks and van der Weide (2003), where social interaction is incorporated in a CBS, in analogy with Brock and Durlauf (2001) who consider social interaction in a dynamic discrete choice setting. 
If we incorporate the dynamics for the average belief $\mu_{t}$, which is given in Eq. (8), it follows that the market's price dynamics is described by the following ARIMA model:

$$
x_{t}=x_{t-1}+\frac{J}{J+1} \sigma\left[u_{t}-\alpha u_{t-1}\right] .
$$

The amount of endogenous uncertainty entering the price dynamics is proportional to $J /(J+1)$. Since this function is increasing in $J$ and takes values between 0 and 1 , it is convenient to define a measure of herding $\lambda$, via

$$
\lambda \equiv \frac{J}{J+1},
$$

so that the price dynamics in the absence of news can be concisely stated as

$$
\Delta x_{t}=\sigma \lambda\left(u_{t}-\alpha u_{t-1}\right),
$$

where $\Delta x_{t} \equiv x_{t}-x_{t-1}$. As herd behaviour acts to affect the effective number of agents, it should come as no surprise that a change in the extent of herding $\lambda$ can not be distinguished from a reduction of the number of agents in the CBS. Only their joint effect can be identified. ${ }^{7}$

At this stage all stochasticity in the model can be associated with endogenous uncertainty. That is, the randomness observed by the econometrician reflects the random nature with which individual agents select beliefs from the distribution of beliefs. If in addition we include independent, identically distributed (IID) exogenous shocks $\xi_{t}$ to the system, we find:

$$
\begin{aligned}
\Delta x_{t} & =\sigma \lambda\left(u_{t}-\alpha u_{t-1}\right)+\rho \xi_{t} \\
& \sim \eta\left(u_{t}^{\prime}-\psi u_{t-1}^{\prime}\right),
\end{aligned}
$$

where $\left\{u_{t}^{\prime}\right\}$ is a sequence of IID standard normal random variables, $\eta$ a scaling parameter, and $-\psi$ a moving average coefficient. The relation between our original parameters $(\sigma, \lambda, \alpha, \rho)$ and the new parameters $(\eta, \psi)$ is the following:

$$
\begin{aligned}
& \eta=\left(\sigma^{2} \lambda^{2}+\rho^{2}\right)^{\frac{1}{2}} \\
& \psi=\frac{\alpha \sigma \lambda}{\left(\sigma^{2} \lambda^{2}+\rho^{2}\right)^{\frac{1}{2}}} .
\end{aligned}
$$

The equivalence of the two representations in Eq. (14) follows directly from comparing the auto-covariance functions of both representations. While both representations

\footnotetext{
${ }^{7}$ Diks and van der Weide (2003) related the amount of endogenous noise to both the market impact of agents, and the correlation between agent's decisions. The effective number of agents (which is inversely proportional to the variance of endogenous noise) is given by $n_{\text {eff }}=$ $\left(\sum_{i=1}^{n} w_{i}^{2}+\rho \sum_{i} \sum_{j \neq i} w_{i} w_{j}\right)$ where $n$ is the total number of agents, $\left\{w_{i}\right\}$ represent their market impacts, and where $\rho$ is the correlation between agent's beliefs. Taking equal weights, $w_{i}=\frac{1}{n}$, and letting $n$ tend to infinity as we do here, only leaves $n_{\mathrm{eff}}=\rho^{-1}$.
} 
describe the same ARIMA $(0,1,1)$ model, the reduction from four to two parameters shows that the first representation was over-parameterized. The original (behavioral) model parameters can therefore not be identified all independently. For an introduction to ARIMA and related models we refer to Mills (1999).

It is worth noting that we have established a link between behavioral finance and econometric modelling. As a direct result, one can associate the parameters from the econometric model with economically relevant and/or behavioral quantities. In this particular example, the scale of the endogenous noise appears in the econometric model as the product $\sigma \lambda$ of the dispersion $\sigma$ of the beliefs distribution and the herding parameter $\lambda$, while $\rho$ measures the size of the exogenous noise.

\subsection{A-synchronous updating}

So far, as is also common in economic dynamic models based on discrete choice, we have assumed implicitly that the time scale on which agents re-evaluate strategies is short with respect to the trading time. It seems reasonable to assume that in the absence of costs for evaluating and updating beliefs, every agent will indeed decide to update every period. However, if updating is either time consuming or costly, agents are likely to update less frequently. Hong et al. (2000) found empirical evidence supporting this view. In their empirical study they concluded that inexperienced analysts revise their forecasts more frequently than experienced analysts.

In general, if the time scales on which agents review strategies is longer in practice than the clock rate at which prices/exchange rates are quoted, the dynamics of both prices and the distribution of beliefs will be affected. Indeed, Brock and LeBaron (1996) find that time series with more realistic properties, such as strong persistence of volatility, are obtained in the case of less frequent updating. The effects of the time scale for evaluations can be incorporated in the model in several ways. One might take the approach of Brock and LeBaron (1996) where all agents update their beliefs all at once with a certain fixed probability per time unit.

An alternative approach, which we will follow here, is to assign a probability $\delta$ that individual agents do not update their beliefs, and a probability $1-\delta$ that individual agents do update their belief, at each time step. The latter is generally more difficult to implement, since it implies that the beliefs distribution becomes a mixture with weight $\delta$ on the old utility function, and $1-\delta$ on the new, that is,

$$
\begin{aligned}
& \phi_{t}(\theta)=\delta \phi_{t-1}(\theta)+(1-\delta) \tilde{\phi}_{t}(\theta) \\
& \tilde{\phi}_{t}(\theta) \sim e^{\beta U_{t}(\theta)} \sim e^{\beta(1-\alpha) \pi_{t}(\theta)}\left[\tilde{\phi}_{t}(\theta)\right]^{1-\alpha} .
\end{aligned}
$$

Here $\tilde{\phi}_{t}(\theta)$ represents the up-to-date distribution of choices that would be made based on information up to the recent past, while the actual beliefs distribution $\phi_{t}(\theta)$ is still partly based on old information. In simple cases, however, where the mean and variance of the mixture depend on the past utility only through the previous mean and 
variance, a closed form updating rule for the new mean and variance can still be given. This is demonstrated in the following example.

The average belief and the dispersion of beliefs among the agents that have decided to update are denoted by $\left\{\mu_{t}, \sigma_{t}^{2}\right\}$, where $\sigma_{t}^{2}=\sigma^{2}$ is still constant over time. The average and the dispersion of beliefs for the entire population is now denoted by $\mu_{\delta, t}$ and $\sigma_{\delta, t}^{2}$. Using Eq.(16) it can be verified that the dynamics of the first two moments of the beliefs distribution is now governed by:

$$
\begin{aligned}
\mu_{\delta, t} & =\delta \mu_{\delta, t-1}+(1-\delta) \mu_{t} \\
\left(\mu_{\delta, t}^{2}+\sigma_{\delta, t}^{2}\right) & =\delta\left(\mu_{\delta, t-1}^{2}+\sigma_{\delta, t-1}^{2}\right)+(1-\delta)\left(\mu_{t}^{2}+\sigma^{2}\right),
\end{aligned}
$$

where as before (Eq. 8), we have:

$$
\mu_{t}=\alpha \mu_{t-1}+(1-\alpha) x_{t} .
$$

If we take squares on both sides of the first relation in Eq. (17) and subtract both relations to eliminate $\mu_{\delta, t}^{2}$, we find the following dynamics for the dispersion of beliefs $\sigma_{\delta, t}^{2}$ :

$$
\sigma_{\delta, t}^{2}=\sigma^{2}+\delta\left(\sigma_{\delta, t-1}^{2}-\sigma^{2}\right)+\delta(1-\delta)\left(\mu_{t}-\mu_{\delta, t-1}\right)^{2} .
$$

Thus the dispersion of beliefs exhibits fluctuations when agents do not move together, even when the dispersion of beliefs among those who adapt is constant in each period. The movements are driven by the differences in average beliefs between those who decide to adapt and those who wait.

The time evolution for the price is now described by the following analogue of Eq. (12):

$$
x_{t}=\mu_{\delta, t-1}+\lambda \sigma_{\delta, t-1} u_{t},
$$

where $\lambda$ is our measure for herding and $\sigma_{\delta, t-1}$ is the time varying standard deviation of the beliefs distribution. Let us first focus on the average beliefs $\left\{\mu_{t}, \mu_{\delta, t}\right\}$. Note that Eqs (17) and (18) give, respectively,

$$
(1-\delta) \mu_{t}=\mu_{\delta, t}-\delta \mu_{\delta, t-1}
$$

and

$$
(1-\delta) \mu_{t}=\alpha(1-\delta) \mu_{t-1}+(1-\alpha)(1-\delta) x_{t},
$$

which together yield:

$$
\mu_{\delta, t}-\delta \mu_{\delta, t-1}=\alpha\left(\mu_{\delta, t-1}-\delta \mu_{\delta, t-2}\right)+(1-\alpha)(1-\delta) x_{t} .
$$

If we substitute $\mu_{\delta, t}=x_{t+1}-\left(\lambda \sigma_{\delta, t} u_{t+1}\right)$ (Eq. 20), and rearrange terms, we obtain:

$$
\Delta x_{t}=\alpha \delta \Delta x_{t-1}+\lambda\left(\sigma_{\delta, t-1} u_{t}-(\alpha+\delta) \sigma_{\delta, t-2} u_{t-1}+\alpha \delta \sigma_{\delta, t-3} u_{t-2}\right),
$$

where, as before, $\left\{u_{t}\right\}$, is again a sequence of IID standard normal random variables. 
For positive memory $(\alpha>0)$ and a-synchronous updating $(\delta>0)$, the dynamics of the differences $\left\{\Delta x_{t}\right\}$ is described by an ARMA $(1,2)$ process. Interestingly, and in contrast with our previous example, the (conditional) variance of the disturbances is no longer constant. The conditional variance is proportional to the dispersion of beliefs, which now exhibits endogenous fluctuations. Intuitively, these fluctuations in the degree of heterogeneity can be ascribed to the differences in opinion between those who decide to update their beliefs and those who believe that updating can wait. According to Eq. (19), the dynamics of heterogeneity is described by an autoregressive process of order one, from which one may correctly expect a GARCH $(1,1)$ model to emerge for the conditional variances. For clarity of exposition we set memory to zero i.e. $\alpha=0$, so that the initial $\operatorname{ARMA}(1,2)$ process for $\left\{\Delta x_{t}\right\}$ is stripped down to a MA(1) process:

$$
\begin{aligned}
\Delta x_{t} & =\lambda \sigma_{\delta, t-1} u_{t}-\lambda \delta \sigma_{\delta, t-2} u_{t-1} \\
& \equiv \epsilon_{t}-\delta \epsilon_{t-1}
\end{aligned}
$$

where the conditional variance $h_{t}=\operatorname{Var}_{t}\left(\epsilon_{t}\right)=\lambda^{2} \sigma_{\delta, t-1}^{2}$ is updated according to:

$$
h_{t}=(1-\delta) \lambda^{2} \sigma^{2}+\delta(1-\delta) \lambda^{2} \epsilon_{t-1}^{2}+\delta h_{t-1},
$$

where we have used Eq. (19) together with the fact that for $\alpha=0$, Eq. (18) implies $\mu_{t}=x_{t}$, so that Eq. (20) gives $\mu_{t}-\mu_{\delta, t-1}=x_{t}-\mu_{\delta, t-1}=\epsilon_{t-1}^{2}$. Because $0<\lambda^{2}<1$ a given GARCH parameter $\delta$ implies an upper limit of $\delta(1-\delta)$ for the ARCH parameter. Note that the sum $s=\delta+\lambda^{2} \delta(1-\delta)$ of the ARCH and GARCH parameters satisfies $0<s<1$ for $0<\delta<1$, so that the resulting GARCH process is stable.

\subsection{Heterogeneity in memory}

In this section we consider the third and final behavioral extension of the benchmark model: heterogeneity in memory. As the diversity in future time horizons seems relevant, also the diversity in past time horizons may play an important role in the coevolution of prices and beliefs. With past time horizons we refer to the history of price observations used to test candidates for future trading. Agents who are more concerned with price behaviour on the short-term are more likely to compare performances over a smaller sample of recent observations than agents who have a clear interest in the long-term dynamics. Another reason for agents to use small memory parameters might be that they believe that the world they live in is inherently nonstationary, and only recent performances can be considered relevant.

Recently, a number of studies addressed the issue of different (future) time horizons among agents (see e.g. Muller et al., 1997; Dacorogna, 2002). This generalization is supported by the observation that agents trade at different frequencies. That is, the population of traders often consists of both long-term traders and short-term traders. Heterogeneity in agents' time scale are believed to be responsible for a number of stylized facts. Long term traders naturally focus on long-term behavior of prices thereby neglecting fluctuations at the smallest time scale, whereas short-term traders are not 
concerned with price movements on the long-run but rather aim to exploit short term predictability. The effects of the diversity in time horizons on price dynamics have been studied by LeBaron (2001) in an artificial stock market model. He concluded that the presence of heterogeneity in horizons may lead to an increase in return variability, as well as volatility-volume relationships similar to those of actual markets. Dacorogna (2002) found evidence supporting trading horizon heterogeneity to be responsible for the slow decay of correlations found empirically.

In the case where the memory parameter $\alpha$ is distributed among agents according to the $\operatorname{pdf} \nu(\alpha)$ with $0<\alpha<1$, the most recent beliefs distribution for each memory type can be denoted by $\tilde{\phi}_{\alpha, t}(\theta)$. The overall beliefs distribution is then given by

$$
\phi_{t}(\theta)=\int_{0}^{1} \tilde{\phi}_{\alpha, t}(\theta) \nu(\alpha) \mathrm{d} \alpha .
$$

Again, in general it can be very difficult to derive the updating rules for $\phi_{t}(\theta)$ analytically. However, if closed form expressions for the moments $E\left[\alpha^{k}\right]$ with respect to $\nu(\alpha)$ are known, for example, it is possible to derive analytic expressions for some of the moments, such as the mean $\mu_{t}$ and variance $\sigma_{t}^{2}$, of the beliefs distribution. This is demonstrated in some examples below.

Let $\alpha_{i}$ denote the memory of agent $i$, and $\mu_{i, t}$ and $\sigma_{i, t}^{2}$ represent the first two moments of the beliefs distribution corresponding to agents with memory parameter $\alpha_{i}$. In the case of synchronous updating, it can be verified that $\sigma_{i, t}^{2}=\sigma^{2}$ for all $i$, and that the dynamics of the average $\left\{\mu_{t}\right\}$ over the entire population is described by:

$$
\mu_{t}=\int_{0}^{1} \mu_{t}(\alpha) \nu(\alpha) \mathrm{d} \alpha .
$$

For the mean over agents with memory parameter $\alpha_{i}$ we find

$$
\begin{aligned}
\mu_{i, t} & =\alpha_{i} \mu_{i, t-1}+\left(1-\alpha_{i}\right) x_{t} \\
& =\left(1-\alpha_{i}\right) \sum_{k=0}^{\infty} \alpha_{i}^{k} x_{t-k} \\
& =\sum_{k=0}^{\infty} \alpha_{i}^{k} x_{t-k}-\sum_{k=0}^{\infty} \alpha_{i}^{k+1} x_{t-k} .
\end{aligned}
$$

Taking expectations on both sides yields:

$$
\mu_{t}=\sum_{k=0}^{\infty} E_{\nu}\left[\alpha^{k}\right] x_{t-k}-\sum_{k=0}^{\infty} E_{\nu}\left[\alpha^{k+1}\right] x_{t-k},
$$

where $E_{\nu}[\cdot]$ stands for the expectation with respect to the distribution of the memory parameter among agents, which has pdf $\nu(\alpha)$. For a large but finite number of agents, endogenous noise enters the price equation via

$$
x_{t}=\mu_{t-1}+\sigma_{t} \lambda u_{t}
$$


where $\lambda$ is a measure for the scale of endogenous noise which can either result from the presence of herding or a finite number of agents, and $\left\{u_{t}\right\}$ a sequence of independent random variables with mean zero and unit variance (cf. Eqs (12) and (20)). From Eqs (24) and (25) we obtain:

$$
\begin{aligned}
x_{t} & =\sum_{k=0}^{\infty} E_{\nu}\left[\alpha^{k}\right] x_{t-k-1}-\sum_{k=0}^{\infty} E_{\nu}\left[\alpha^{k+1}\right] x_{t-k-1}+\sigma_{t} \lambda u_{t} \\
& =x_{t-1}-\sum_{k=1}^{\infty} E_{\nu}\left[\alpha^{k}\right]\left(x_{t-k}-x_{t-k-1}\right)+\sigma_{t} \lambda u_{t} .
\end{aligned}
$$

It follows that the increments $\Delta x_{t}=x_{t}-x_{t-1}$ are described by:

$$
\Delta x_{t}=-\sum_{k=1}^{\infty} E_{\nu}\left[\alpha^{k}\right] \Delta x_{t-k}+\sigma_{t} \lambda u_{t} .
$$

Upon defining $L$ as the lag operator i.e. $L x_{t}=x_{t-1}$, we can reformulate to obtain:

$$
\left(\sum_{k=0}^{\infty} E_{\nu}\left[\alpha^{k}\right] L^{k}\right) \Delta x_{t}=\sigma_{t} \lambda u_{t} .
$$

If $\alpha$ is assumed to be uniformly distributed on $[0,1]$, then we have $E_{\nu}\left[\alpha^{k}\right]=\frac{1}{1+k}$ (see e.g. Linden, 1999, who studies the aggregated AR(1) process with uniformly distributed coefficients). As the latter does not decay exponentially when $k$ tends to infinity, we observe long memory behavior for $\left\{\Delta x_{t}\right\}$. Thus for $\alpha \sim \operatorname{UNIF}(0,1)$, we have:

$$
\left(\sum_{k=0}^{\infty} \frac{L^{k+1}}{k+1}\right) \Delta x_{t}=L \sigma_{t} \lambda u_{t}
$$

or equivalently:

$$
-\ln (1-L) \Delta x_{t}=\sigma_{t-1} \lambda u_{t-1}
$$

where the operator $\ln (1-L)$ on the left hand side should be interpreted in terms of its power series in $L$.

More generally, we may consider a memory parameter distributed according to a $\operatorname{BETA}(a, b)$ distribution on $(0,1)$, with pdf

$$
\nu(\alpha)=\frac{\Gamma(a+b)}{\Gamma(a) \Gamma(b)} \alpha^{a-1}(1-\alpha)^{b-1}, \quad 0<\alpha<1 .
$$

The expectation $E_{\nu}\left[\alpha^{k}\right]$ can then be expressed as

$$
E_{\nu}\left[\alpha^{k}\right]=\frac{\Gamma(a+b)}{\Gamma(a)} \frac{\Gamma(a+k)}{\Gamma(a+b+k)} .
$$


Although we will leave a detailed analysis of the dynamics for some of these more general cases for future research, we will make some remarks here.

For $a=d, b=1-d$, with $d \in(0,1)$, one finds

$$
E_{\nu}\left[\alpha^{k}\right] L^{k}=\frac{\Gamma(k+d)}{\Gamma(d) \Gamma(k+1)} L^{k} .
$$

Since these correspond to the terms of a Taylor expansion of $(1-L)^{-d}$ around $L=0$, the sum in Eq. (26) can be written in terms of the following fractionally integrated process:

$$
(1-L)^{-d} \Delta x_{t}=\sigma_{t} \lambda u_{t} .
$$

Similar processes, when driven by IID normal innovations, are known as fractionally integrated brownian motion. For $d \in\left(0, \frac{1}{2}\right)$ the latter is invertible and stationary, and the autocorrelation coefficients are negative (see Hosking, 1981), a property which is commonly observed in exchange rate returns, for example. Although Hosking refers to these processes as being short-memory, both the autocorrelation function and partial autocorrelation function decay hyperbolically to zero. Therefore fractionally integrated brownian motion is often referred to as being long-range dependent (see e.g. Campbell et al., 1997). The fact that we find a related process is in line with the results of Granger (1980), who showed that aggregation of simple processes with different characteristic time scales can easily lead to long memory.

However, considering $\sigma_{t}$ to be time independent would be an oversimplification. Although the variance $\sigma_{t}^{2}$ of beliefs among agents using the same memory parameter $\alpha$ converges to a constant $\sigma^{2}$, the belief parameters $\theta_{t}$ for agents with different memory parameters $\alpha$ are centered around different values of $\mu_{t}$, which depend on $\alpha$. The conditional variance of the $\theta_{t}$ for the population as a whole is therefore given by

$$
\sigma_{t}^{2}=\sigma^{2}+\operatorname{Var}_{\nu}\left(\mu_{t}(\alpha)\right)
$$

where the variance of $\mu_{t}(\alpha)$ is taken with respect to the distribution $\nu(\alpha)$ of the memory parameter $\alpha$ among the population. For this variance one finds

$$
\begin{aligned}
\operatorname{Var}_{\nu}\left(\mu_{t}(\alpha)\right) & =\operatorname{Var}_{\nu}\left((1-\alpha) \sum_{k=0}^{\infty} \alpha^{k} x_{t-k}\right) \\
& =E_{\nu}\left[\left((1-\alpha) \sum_{k=0}^{\infty} \alpha^{k} x_{t-k}\right)^{2}\right]-\mu_{t}^{2},
\end{aligned}
$$

where, as before, $\mu_{t}$ is given by Eq. (23). The first term on the right hand side can alternatively be expressed as

$$
E_{\nu}\left[\left((1-\alpha) \sum_{k=0}^{\infty} \alpha^{k} x_{t-k}\right)^{2}\right]=E_{\nu}\left[(1-\alpha)^{2} \sum_{m=0}^{\infty} \alpha^{m} \sum_{n=0}^{m} x_{t-n} x_{t-m+n}\right] .
$$

Although this is a closed form expression of the second moment of $\mu_{t}\left(\alpha_{i}\right)$ with respect to $\nu(\alpha)$, it seems that this term cannot be expressed in a form which permits simple 
recursive updating as new prices $x_{t}$ become available. It does however show that the dispersion of beliefs among agents obeys a non-trivial time dependence in the presence of heterogeneity in memory, and that the second moment of the innovations governing the price dynamics will exhibit a likewise complexity.

\section{Empirical validation}

As a framework for modelling the stochastic dynamics of prices and beliefs explicitly, the CBS aims to make insightful how the stylized facts of observed prices may be traced back to the unobservable diversity of beliefs, and vice versa. In the previous section we examined the specific role of three different behavioural aspects. By keeping the basic CBS as simple as possible, the three extended behavioral models were shown to relate directly to familiar econometric time-series models. The basis of the models was found to be the ARIMA model. Interestingly, a-synchronous updating of beliefs was found to translate into GARCH-type structure, and long range dependence (or long memory) emerged for agents employing different time horizons in evaluating past performance of beliefs (i.e. heterogeneity in memory).

As an immediate result, we find that the parameters of these well-known models from econometrics suddenly have an economic and behavioral interpretations attached. Although tempting, interpreting these hidden behavioral parameters directly from the data seems optimistic in this stage. We are aware of the fact that several relevant behavioral model aspects and economic variables have been ignored. The addition of relevant other features to the model is likely to affect the GARCH structure, or to alter the long range dependence. Our models do not capture, for example, longer range effects as described by Taylor and Allen (1992). Note, however, that the idea that the degree of fractional integration is a proxy for the degree of heterogeneity in memory finds support in Dacorogna (2002).

In this light there is little interest in focusing on the exact values of the parameter estimates. However, it will be interesting to see whether the signs of the MA coefficients and roughly the size of the GARCH parameters are both consistent with what is predicted by the theory i.e. the stylized CBSes. Finding negative values for the memory parameter, for example, is likely not to win support for our CBS. The predicted effects of the behavioral assumptions are put to the test in this empirical validation. Focus is in particular on the model obtained in the presence of a-synchronous updating (see section 3.2, Eqs. 21 and 22). The ARCH and GARCH coefficients that emerge in that example are given by $\delta(1-\delta)$ and $\delta$, respectively, where $(1-\delta)$ denotes the probability for each agent to update his belief at any given period in time. This behavioural aspect of the model thus predicts a relation between the ARCH and the GARCH coefficients, which can be checked empirically. Moreover, the model predicts a negative MA(1) coefficient equal to $-\delta$. Hereby we have set memory to zero, leaving us with a model that is more tractable, but also with one degree of freedom less. Keep in mind, however, that in the case of herding we also predicted a negative MA(1) coefficient, equal to $-\alpha$ 


\begin{tabular}{lrccc}
\hline \hline Country & MA(1) & ARCH & GARCH & ARCH $_{\max }^{\text {Implied }}$ \\
\hline Belgium & -0.05 & 0.17 & 0.24 & 0.18 \\
Germany & 0.00 & 0.15 & 0.60 & 0.24 \\
Finland & -0.05 & 0.05 & 0.95 & 0.05 \\
France & -0.03 & 0.05 & 0.91 & 0.08 \\
Austria & -0.03 & 0.06 & 0.90 & 0.09 \\
Singapore & -0.08 & 0.08 & 0.91 & 0.08 \\
\hline \hline
\end{tabular}

Table 1: Estimates of an MA(1) model with $G A R C H(1,1)$ disturbances for six exchanges rate returns, and implied ARCH upper limit (last column). The predicted MA coefficient was $-\psi$ with $\psi$ non-negative (Eq. 15), implying non-positive MA coefficients, as found in all cases.

(see Eq. 13).

The data used to illustrate the model are quotes from the foreign exchange market. We consider the daily exchange rate of the US dollar against the local currencies of six different countries. Five of these countries are European: Belgium, Germany, Finland, France, and Austria, while the last is from South East Asia, namely Singapore. Let $p_{t}$ denote the amount of US dollars one can buy for one unit of the local currency at time $t$. Instead of absolute prices, however, we consider the daily logarithms i.e. $x_{t}=\log p_{t}$, so that $\Delta x_{t}$ denotes the daily log return on the exchange rate. The sample period, from 5-11-1987 until 5-11-2002, includes a total of 3914 observations.

Table 1 reports the estimates of an MA(1) model with $\mathrm{GARCH}(1,1)$ disturbances for all six of the exchanges rate returns. The first three columns list the actual estimates, whereas the fourth column reports the implied upper limit for the ARCH coefficient i.e. the upper limit implied by our behavioral model which predicts a relation between the ARCH and GARCH coefficients (see Eq. 22, and the discussion following it). According to the latter equation, a GARCH coefficient of $\delta$ implies an ARCH coefficient no larger than $\delta(1-\delta)$. A first look at these results shows that the size of the estimated ARCH effect indeed satisfies this criterion obtained in the case of a-synchronous updating of beliefs. With only one degree of freedom, namely the extent to which beliefs are updated in an a-synchronous fashion, it should not be surprising that the exact size of the estimated MA coefficient is not consistent with the (G)ARCH parameters. Apart from having set $\alpha$ to zero, the setup is also stylized in other respects. With that in mind, it is encouraging to see that the signs of the estimated MA coefficient are also consistent with what we would expect from the theory. In sum, in light of all limitations, we consider the empirical validation successful and hope that it may open doors to more informative empirical assessments by means of more sophisticated CBSes.

\section{Concluding remarks}

The purpose of this paper was to make insightful how certain behavioral features may render some of the well-established stylized facts of observed prices. In order of treat- 
ment, we considered: (i) herd behaviour; (ii) a-synchronous updating of beliefs; and (iii) heterogeneity in the time horizons (memory) of agents. The adopted framework is that of the Continuous Beliefs System (CBS) (see Diks and van der Weide, 2003). We used two qualities of a CBS that are particularly useful. First, it enables modelling the evolution of the distribution of beliefs in an explicit fashion. Second, it does so by relating the unpredictable nature of prices with that of the unpredictable nature of individual preferences. In sum, we used the fact that a CBS allows for describing the stochastic dynamics of both prices and beliefs explicitly by means of a Random Dynamical System.

By keeping our benchmark CBS illustrative but deliberately simple, the stochastic dynamics of each of the three behavioral models was shown to correspond directly to a model well-known from time-series econometrics. We came across ARIMA, ARFIMA, and GARCH. This marks our first result, as the stylized facts for which these models are celebrated may now be traced back to the hidden features of beliefs which we hold responsible. Here, the ultimate simplicity of the benchmark CBS helped us to isolate the role of each of the three behavioural features considered.

As we summarize the results for the three models seperately, keep in mind that our point of departure was a model in which the degree of heterogeneity is constant, and where price dynamics is described by a random walk. For each behavioral extension we investigated how it modifies both the dynamics of prices and that of heterogeneity.

The effect of herd behaviour is roughly that of reducing the number of agents in the market. As such, it increases the amount of endogenous uncertainty, and thereby the level of volatility. Moreover, it adds moving average structure, leading to an ARIMA specification in levels or MA in differences. Notably, it does not alter the dynamics of heterogeneity. The latter remains constant.

Fluctuations do enter the dynamics of heterogeneity when we allow agents to update their beliefs at different frequencies. These fluctuations are found to be driven by the differences in average beliefs between those who decide to evaluate and update, and those who believe that updating can wait. If endogenous uncertainty persists in the market, for example as a result of herd behaviour, a time-varying degree of heterogeneity results into a time-varying level of volatility. The extent of persistence in volatility is governed by the likelihood that individual agents delay the updating of their beliefs. This result finds support in an earlier study by Brock and LeBaron (1996), who saw the persistence in volatility of simulated price returns rise to a more realistic level when allowing for less frequent updating. Interestingly, we obtained an explicit description of how the updating of beliefs interacts with the volatility process. For our stylized but yet intuitive example we found the dynamics that to be exactly that of a $\operatorname{GARCH}(1,1)$ model.

Heterogeneity in the time horizons (memory) of agents was found to enrich the dynamics of both the first and second moment. Assuming a distribution in memory rather than identical memory for each agent, we encountered one more stylized fact, namely that of long-range dependence. Leaving aside the volatility process for a moment, taking a $\operatorname{BETA}(d, 1-d)$ distribution for memory, we obtained the well- known 
fractionally integrated process documented by e.g. Hosking (1981) for the first moment. When delving into greater detail, we quickly stumbled upon greater complexity. In particular, the degree of heterogeneity was found to move in mysterious ways. Although the heterogeneity among those who employ identical memory is constant over time, additional dispersion of opinions is present which can be attributed exclusively to the fact that not all agents operate at the same time horizon. The latter does not only add to the overall degree of heterogeneity, it was also found to be time-varying.

To understand the complexity, note that the supply of fluctuations is provided by the dispersion of average beliefs across groups of agents that are operating at different time horizons. As memory plays an important role in the updating of beliefs, we find that the evolution of average beliefs for each of those groups of agents interacts differently with the price dynamics, given that their memories are different. Heterogeneity being driven by the diversity of these evolutions, it requires aggregation of these different dynamics to obtain the dynamics of heterogeneity. The latter, although interesting, easily becomes convoluted.

At the end of the paper we included a modest empirical validation to address the intimate relation between our behavioural models and the more familiar models from econometrics. As a corollary, the parameters of these time-series models now have an economic and/or behavioural interpretation attached. For example, our stylized model predicts a relation between the ARCH and GARCH parameters. Merely as a 'reality check', we verified whether both that relation, and the signs of the other coefficients, finds support in the empirical data. In light of all simplifications, we consider the empirical validation successful, and hope that more informative empirical assessments by means of more sophisticated CBSes can be made in the future.

As a final remark we would like to comment on the difference with the recently proposed LTL approach of Brock et al. (2003). Although a CBS is more explicit in terms of the beliefs distribution, the price dynamics of a CBS and an LTL are closely related. (For a more elaborate comparison, see Diks and van der Weide (2003).) In case the opportunity function $\varphi(\theta)$ (see footnote 2) is normalizable, the LTL coincides with the deterministic part of the corresponding CBS. However, there are two key differences. For one, it is not clear how an LTL can be set up with a non-normalizable opportunity function (which for an LTL corresponds to the pdf according to which strategies are drawn at random). More importantly, an LTL is a deterministic dynamical system by construction, and up until now not tailored to dealing with endogenous randomness. It is exactly the associated stochastic dynamics that plays a leading role in this paper.

\section{References}

Avery, C. and Zemsky, P. (1998). Multidimensional uncertainty and herd behavior in financial markets. American Economic Review, 88, 724-748.

Bikhchandani, S., Hirschleifer, D. and Welch, I. (1992). A theory of fads, fashion, cus- 
tom and cultural change as informational cascases. Journal of Political Economy, 100, 992-1027.

Brock, W. A. and Durlauf, S. N. (2001). Discrete choice with social interactions. Review of Economic Studies, 68, 235-260.

Brock, W. A. and Hommes, C. H. (1998). Heterogeneous beliefs and routes to chaos in a simple asset pricing model. Journal of Economic Dynamics and Control, 22, 1235-1274.

Brock, W. A., Hommes, C. H. and Wagener, F. O. O. (2003). Evolutionary dynamics in markets with many trader types. Technical Report. CeNDEF Working paper 02-10, University of Amsterdam. Journal of Mathematical Economics, forthcoming.

Brock, W. A. and LeBaron, B. (1996). A dynamic structural model for stock return volatility and trading volume. Review of Economics and Statistics, 78, 94-110.

Brock, W.A. and Hommes, C.H. (1997). Rational routes to randomness. Econometrica, 65, 1059-1095.

Campbell, J. Y., Lo, A. W. and MacKinley, A. C. (1997). The Econometrics of Financial Markets. Princeton University Press, Princeton, NJ.

Dacorogna, M. (2002). Scaling analysis to characterize financial markets. Technical Report. Paper presented at the CeNDEF workshop, Leiden, The Netherlands, June 2002. See http://www.fee.uva.nl/cendef.

Diks, C. and van der Weide, R. (2003). Heterogeneity as a natural source of randomness. Technical Report. CeNDEF working paper 03-05. University of Amsterdam. Tinbergen Institute Discussion Paper 2003-073/1.

Frankel, J. A. and Froot, K. A. (1988). Chartists, fundamentalists and the demand for dollars. Greek Economic Review, 10, 49-102.

Frankel, J. A. and Froot, K. A. (1990). Chartists, fundamentalists and trading in the foreign-exchange market. American Economic Review, 80, 181-185.

Froot, K. A., Scharfstein, D. S. and Stein, J. (1992). Herd on the street: Informational inefficiencies in a market with short-term speculation. Journal of Finance, 47, 14611484 .

Granger, C. (1980). Long memory relationships and the aggregation of dynamic models. Journal of Econometrics, 14, 227-338.

Hommes, C. (2001). Financial markets as nonlinear adaptive evolutionary systems. Quantitative Finance, 1, 149-167. 
Hong, H., Kubik, J. D. and Solomon, A. (2000). Security analysts' career concerns and herding of earnings forecasts. RAND Journal of Economics, 31, 121-144.

Hosking, J. R. M. (1981). Fractional differencing. Biometrika, 68, 165-176.

Kandel, E. and Pearson, N. D. (1995). Differential interpretation of public signals and trade in speculative markets. Journal of Political Economy, 4, 831-872.

Kilian, L. and Taylor, M. P. (2001). Why is it so difficult to beat the random walk forecast of exchange rates? Technical Report. ECB Working paper No. 88. European Central Bank.

Kirman, A. (1993). Ants, rationality and recruitment. Quarterly Journal of Economics, 108, 137-156.

LeBaron, B. (1999). Technical trading rule profitability and the foreign exchange intervention. Journal of International Economics, 49, 125-143.

LeBaron, B. (2000). Agent-based computational finance: Suggested readings and early research. Journal of Economic Dynamics and Control, 24, 679-702.

LeBaron, B. (2001). Evolution and time horizons in an agent-based stock market. Macroeconomic Dynamics, 5, 225-254.

LeBaron, B., Arthur, W. B. and Palmer, R (1999). Time series properties of an artificial stock market. Journal of Economic Dynamics and Control, 23, 1487-1516.

Lehmann, B. N. (1991). Asset pricing and intrinsic values. Journal of Monetary Economics, 28, 485-500.

Levich, R. M. and Thomas, L. R. (1993). The significance of technical trading-rule profits in the foreign exchange market: a bootstrap approach. Journal of International Money and Finance, 12, 451-474.

Linden, M. (1999). Time series properties of aggregated AR(1) processes with uniformly distributed coefficients. Economics Letters, 64, 31-36.

Lo, A. W. and MacKinlay, A. C. (1997). A Non-random Walk Down Wall Street. Princeton University Press, Princeton, NJ.

Lux, T. (1995). Herd behaviour, bubbles and crashes. Economic Journal, 105, 881-896.

Malkiel, B. (1990). A Random Walk Down Wall Street. Norton, New York.

Mills, T. (1999). The Econometric Modelling of Financial Time Series, 2nd edn. Cambridge University Press, Cambridge, UK. 
Muller, U. A., Dacorogna, M. M., Dave, R. R., Olsen, R. B., Pictet, O. V. and Weizsacker, J. E. Von (1997). Volatilities of different time resolutions - analyzing the dynamics of market components. Journal of Empirical Finance, 4, 213-239.

Nelson, L. (2002). Persistence and reversal in herd behavior: Theory and application to the decision to go public. Review of Financial Studies, 15, 65-95.

Scharfstein, D. S. and Stein, J. (1990). Herd behavior and investment. American Economic Review, 80, 465-479.

Sweeney, R. J. (1986). Beating the foreign exchange market. Journal of Finance, 41, 163-182.

Taylor, M. P. and Allen, H. (1992). The use of technical analysis in the foreign exchange market. Journal of Internationl Money and Finance, 11, 304-314.

Trueman, B. (1994). Analysts forecasts and herding behavior. Review of Financial Studies, 7, 97-124. 